\title{
Fluid-Elastic Vibration of Flexible Overflow Weir*
}

\author{
Yuzuru EGUCHI** and Nobukazu TANAKA**
}

\begin{abstract}
The present paper describes the fundamental feature of the fluid-elastic vibration of flexible overflow weir, as observed in the French demonstration fast breeder reactor, Super Phenix-1. In the experimental study, the instability criterion of the fluid-elastic vibration was investigated using a simple experimental apparatus of a rectangular tank separated by a flexible weir. A spring-mass model for sloshing motion of liquid contained in the tank was developed to clarify the mechanism of the instability. The instability condition was analytically derived from the equations of the spring-mass model. The equations of the spring-mass model were also computationally integrated in time to simulate the timewise evolution of the fluid-elastic vibration. The comparison between the experimental and theoretical results indicate that the present theoretical model is capable of predicting the general characteristics of the vibration observed in the experiment. The present study revealed that the lag-time of the water fall at the weir is the most influential parameter on the fluid-elastic instability of a flexible overflow weir.
\end{abstract}

Key Words: Vibration Coupled with Fluid Motion, Self-Excited Vibration, Sloshing, Overflow Weir, Spring-Mass Model

\section{Introduction}

A fluid-elastic vibration of the overflow weir was observed in the French demonstration fast breeder reactor, Super Phenix-1 during the initial operation tests ${ }^{(1)}$. Intensive investigations were initiated by the French research institutes, CEA (Commissariat a l'Energie Atomique) and EdF (Electricité de France) to understand the physical mechanism and to develop a countermeasure for the fluid-elastic vibration. The experimental and theoretical studies identified two types of instability modes : one is related to the sloshing of fluid and the other to the fluid-structure interaction $^{(2) \sim(6)}$. Hisano and Kaneko followed the study using a small-scale co-axial cylinder model and a rectangular model ${ }^{(7)(8)}$. The experimental results showed the two types of instability modes similar to the French experimental results, while potential flow theory was used to explain the instability mechanism. Fukuie and $\mathrm{Hara}^{\left({ }^{(9)}\right.}$ investigated the instability using rectangular tanks and a plane plate supported by a spring, which revealed four types of instability modes exist in the flexible weir system.

* Received 15th December, 1989

* Department of FBR, Central Research Institute of Electric Power Industry, 1646 Abiko, Abiko-shi, Chiba, 270-11, Japan
The geometry of the tank used in the French study was restricted to the plane reservoir or cylindrical thin annulus, which reflected the geometry of the Super Phenix-1. In the present study, on the other hand, the fluid-elastic vibration in a large rectangular tank with a plate was investigated to understand the fundamental feature of the vibration. The experimental apparatus is composed of a rectangular tank and a flexible overflow weir, which is similar to that of Fukuie and $\mathrm{Hara}^{(9)}$ in geometry. The rectangular tank is separated into two reservoir regions (upstream tank and downstream tank) by the overflow weir. The water enters the upstream tank at the bottom and flows over the weir into the downstream tank. The water is discharged from the bottom of the downstream tank and circulated for re-entering the upstream tank. The experimental parameters studied here are the flow rate of water, the rigidity (thickness) of the weir, the water level difference between the two tanks and the aspect ratio of the downstream tank. The time histories of the free surface oscillation of water in the upstream tank and downstream tank were measured by wave height gauges to study the sloshing characteristics. The strain on the weir was also measured by strain gauges to identify the vibrations on the weir.

The motion of the liquid contained in the up- 
stream tank was modeled by the conservation law of fluid. The flexible weir was modeled by the equation for its bending due to the hydrostatic pressure. The sloshing motion of the liquid in the downstream tank was formulated by the simple spring-mass model. The instability condition for this modeled system was derived analytically to identify the relevant parameters for the vibration instability. The numerical calculations of the equations were also carried out to compare the computational with experimental results.

The following section describes the experimental apparatus and the results obtained. The third section is devoted to the description of the spring-mass model developed and to the presentation of the analytical and numerical results. In the fourth section, we discuss the fundamental features of the fluid-elastic unstable vibrations, comparing the experimental and theoretical results. The concluding remarks are given in the final section.

\section{Nomenclature}

\section{$A:$ constant $\quad[\mathrm{m} / \mathrm{kg}]$}

(inversion of effective mass per span)

$c:$ damping coefficient divided by mass $[1 / \mathrm{sec}]$

$D:$ bending rigidity $\left(=E \theta^{3} / 12\left(1-\nu^{2}\right)\right) \quad\left[\mathrm{Pa} \cdot \mathrm{m}^{3}\right]$

$e:$ upstream tank width [m]

$E$ : Young modulus $[\mathrm{Pa}]$

$h(t)$ : overflow height $[\mathrm{m}]$

$\Delta h(t)$ : deviation of $h(t)$ from equilibrium [m]

$g:$ gravitational acceleration $\left[\mathrm{m} / \mathrm{sec}^{2}\right]$

$k$ : spring constant divided by mass $\left(=\omega^{2}\right)$ $\left[1 / \mathrm{sec}^{2}\right]$

$K:$ constant for overflow rate $\left[\mathrm{kg} / \mathrm{m}^{5 / 2} \mathrm{sec}\right]$

$L:$ weir height $[\mathrm{m}]$

$q(t)$ : overflow rate per span length $[\mathrm{kg} / \mathrm{sec} \mathrm{m}]$

$q_{\text {in }}$ : flow rate at inlet per span length $[\mathrm{kg} / \mathrm{sec} \mathrm{m}]$

$Q(t)$ : volume change in the upstream tank by weir bending per span length $\left[\mathrm{m}^{2}\right]$

$s(z)$ : horizontal displacement of weir [m]

$t:$ time [sec]

$\Delta t:$ time increment [sec]

$T$ : period of sloshing [sec]

$V:$ collision velocity of water fall $[\mathrm{m} / \mathrm{sec}]$

$z$ : vertical coordinate $[\mathrm{m}]$

$\beta:$ parameter in Newmark- $\beta$ scheme

$\rho:$ density of fluid $\left[\mathrm{kg} / \mathrm{m}^{3}\right]$

$\rho^{\prime}:$ density of weir $\left[\mathrm{kg} / \mathrm{m}^{3}\right]$

$\eta$ : elevation of sloshing wave in the downstream tank [m]

$\eta^{*}:$ deviation of $\eta$ from equilibrium $[\mathrm{m}]$

$\nu:$ Poisson ratio

$\omega:$ angular frequency $(=2 \pi / T) \quad[1 / \mathrm{sec}]$

$\theta:$ thickness of weir $[\mathrm{m}]$

$\tau:$ lag time of water fall [sec]
()$_{0}$ : equilibrium value or initial value

$\left({ }^{\prime}\right)$ : derivative with respect to time

\section{Experimental Study}

\subsection{Experimental apparatus and procedure}

The experimental apparatus used is described in Fig. 1. As indicated in the figure, the rectangular tank is divided into two regions, upstream tank and downstream tank, by the flexible weir (thin acrylic plate). The water enters the upstream tank at the bottom of the tank and flows over at the top of the flexible weir into the downstream tank. The water is accelerated by gravitational head generated by a header tank, in order to eliminate the vibratory disturbance in the water flow due to the pump. The whole apparatus is installed on the vibration- isolation system to reduce the external vibratory disturbance. The capital letters circled in the figure indicate the locations of the wave gauges to measure the sloshing water elevation. The capital letters wlthout circles indicate the locations of the strain gauges on the flexible weir to measure the vibration strain on the flexible weir.

The experimental parameters studied in this present paper are flow rate, rigidity (thickness) of the flexible weir, water level difference between the upstream tank and downstream tank, and the aspect ratio of the downstream tank. The ranges of the parameters are as follows :

Flow rate $q_{\text {in }}: 1 \mathrm{~kg} / \mathrm{sec}$ to $5 \mathrm{~kg} / \mathrm{sec}$ with $1 \mathrm{~kg} / \mathrm{sec}$ increments.

Thickness of the weir $\theta: 2 \mathrm{~mm}, 4 \mathrm{~mm}, 6 \mathrm{~mm}$ and $15 \mathrm{~mm}$.

Water level difference $\left(L-\eta_{0}\right): 1 \mathrm{~cm}, 2 \mathrm{~cm}, 3 \mathrm{~cm}$,

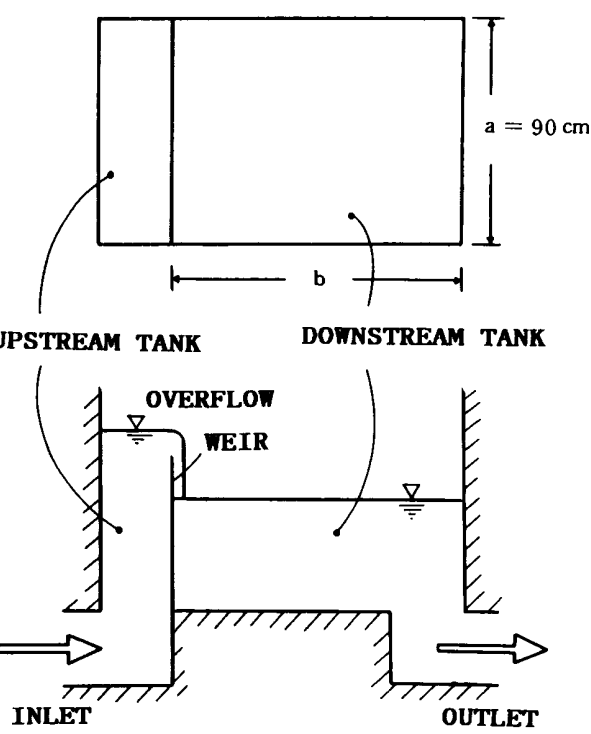

Fig. 1 Configuration of experimental apparatus and location of wave gauges and strain gauges (top : horizontal view, bottom : vertical view) 
$4 \mathrm{~cm}, 5 \mathrm{~cm}$ and $10 \mathrm{~cm}$ maximum

Aspect ratio (b/a): $1.33,0.66$ and 0.33 .

\section{2 Experimental results}

The time histories of the sloshing wave height and the vibrational strain of the weir show that two sloshing modes for the downstream tank and three vibration modes of the flexible weir were observed in the experiment, which are illustrated in Figs. 2 (a) and $(b)$, respectively. The sloshing mode of $(1,0)$ and weir vibration mode of $(1,1)$ are most likely to occur for any experimental case, while the higher modes tend to occur for small weir thickness. Typical timewise developments of downstream-tank sloshing and the weir vibration are shown in Figs. 3 (a) and ( b ). The water level difference significantly influences the magnitude of the fluid-elastic vibration. The peakto-peak values of sloshing water height in the downstream tank at a fully developed stage are plotted against the initial water level difference between the upstream tank and downstream tank in Figs. 4 (a) and (b). These figures indicate that fluid-elastic vibration occurs when the water level difference falls into a specific range, i.e. approximately $1 \mathrm{~cm}$ to $6 \mathrm{~cm}$. This tendency was generally observed for the whole range of the other experimental parameters. The effect of the flow rate on the vibration development was not clearly identified under the experimental conditions, as seen in Figs. 4. The effect of the weir thickness on the fluid-elastic vibration is clear. In the case of the small thickness of $2 \mathrm{~mm}$, higher sloshing modes and higher weir vibration modes were likely to occur, probably due to the imperfectness of the symmetry of the weir installed in the experimental appa-

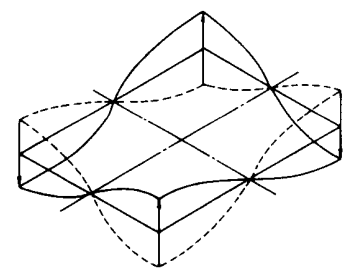

$(1,1)$ MODE

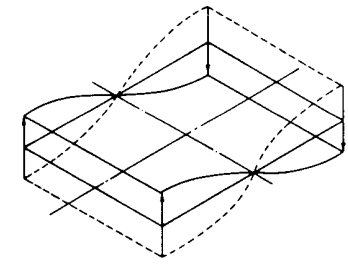

$(1,0)$ MODE (a) Observed sloshing modes in downstream tank

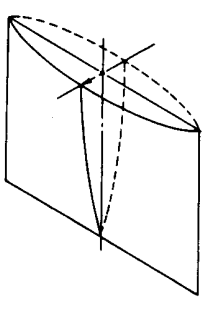

$(1,1)$ MODE

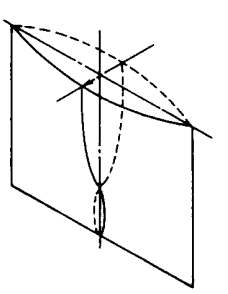

$(1,2)$ MODE

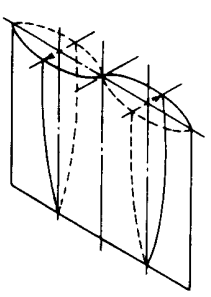

$(2,1)$ MODE (b) Observed vibration modes of weir

Fig. 2 ratus. If the thickness is large, i.e. $15 \mathrm{~mm}$, no vibration was observed in any cases. It was rather difficult to see if there was any influence of the aspect ratio on

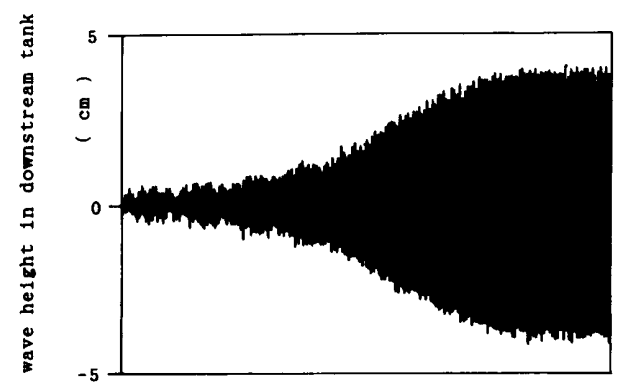

(a) Development of sloshing

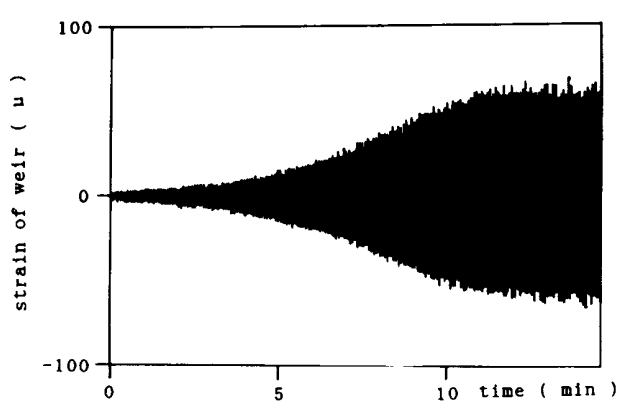

(b) Development of weir vibration

Fig. 3

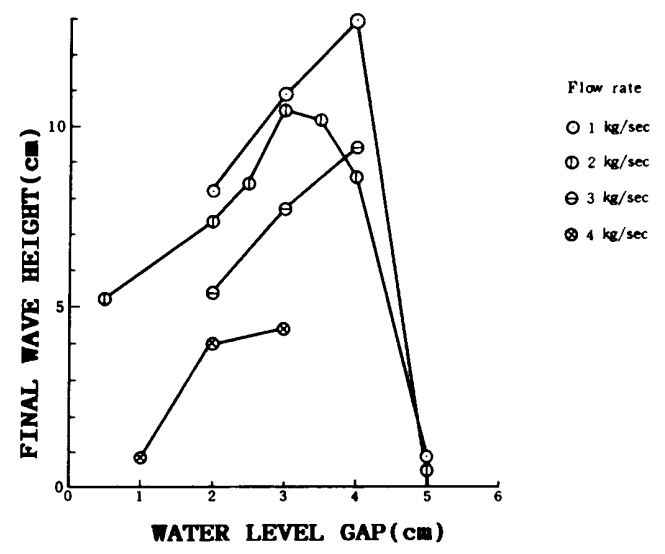

(a) Peak-to-peak value of sloshing height against initial water level gap $(b / a=1.33)$

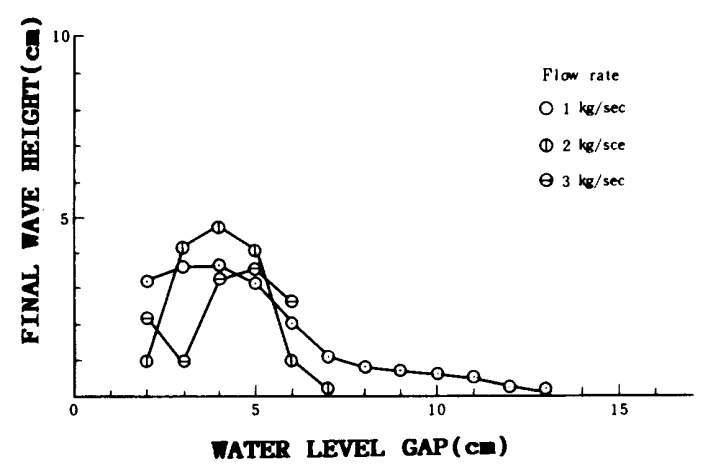

(b) Peak-to-peak value of sloshing height against initial water level gap $(b / a=0.33)$

Fig. 4 
this instability because of the limited number of data. However, it seems to us that the vibration becomes mild as the aspect ratio decreases, as observed by comparing Figs. 4 (a) and (b).

\section{Theoretical Study}

\section{1 Spring-mass model}

The fluid-elastic vibration was modeled by a single-degree-of-freedom system consisting of springmass model : the experimental apparatus is considered to be fully two-dimensional and only the $(1,0)$ sloshing mode and the $(1,1)$ plate vibration mode are considered in the present model. For the upstream tank, the balance of inflow and outflow rates are taken into account; it is noted that the overflow height $h$ is an important variable. The sloshing motion of the free surface in the downstream tank is modeled by a simple spring-mass model excited by the external force produced by the momentum change of the waterfall. The vibration of the weir is formulated by the equation for bending. The displacement of the weir is taken into account in calculating the balance of flow rate in the upstream tank. The schematic illustration of this idealization is given in Fig. 5 .

First, the equation of motion for this mass-spring model is given as follows:

$$
\ddot{\eta}^{*}+c \dot{\eta}^{*}+k \eta^{*}=f(t)
$$

where $\eta$ and $f(t)$ are defined as

$$
\begin{aligned}
& \eta^{*}=\eta-\eta_{0}, \\
& f(t)=-A\left(q(t-\tau) V(t)-q\left(t_{0}\right) V\left(t_{0}\right)\right),
\end{aligned}
$$
and

$q(t)=K h(t)^{3 / 2}$

Note that the definition of each symbol is given in the nomenclature. Equation (1) describes the sloshing behavior in terms of the sloshing wave height $\eta^{*}$ evaluated at the end of the downstream tank. The external force $f(t)$ is given by Eq. ( 3 ) where the positive constant $A$ is physically equivalent to the inverse of the effective mass of the sloshing liquid in

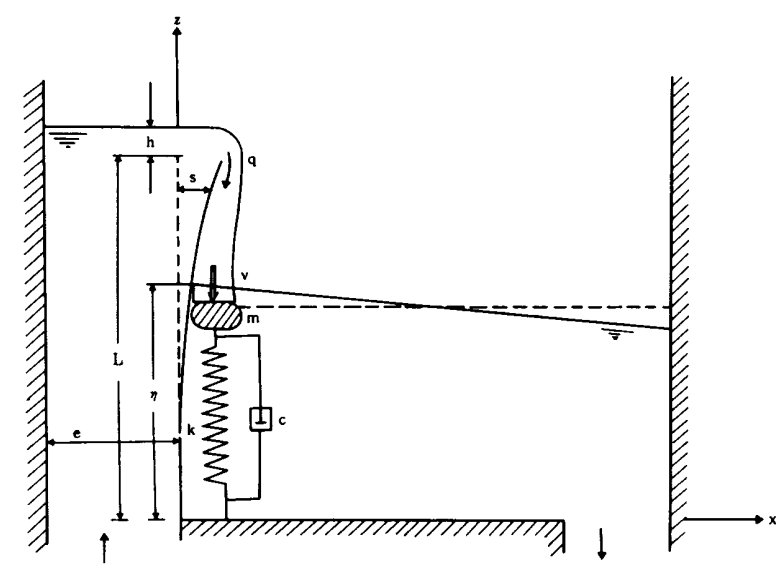

Fig. 5 Spring-mass model idealization the system. The lag-time $\tau$ is taken into account in the model because of the traveling time of water from the top of the weir to the free-water surface in the downstream tank. Although the velocity of water at the free surface, $V(t)$, is obviously a function of time, we assume that $V(t)$ is constant for simplicity. Equation $(4)$ is the empirical relation which connects the overflow height $h(t)$ to the overflow rate $q(t)$.

The balance of the flow rates in the upstream tank is given in the following equation:

$$
\rho e \dot{h}+q(t)+\rho \dot{Q}(t)=q_{\mathrm{ln}},
$$

where the first term in the left-hand side represents the flow rate of the change in the free surface height in the upstream tank : the second term is the overflow rate over the weir, and the third term is the flow rate due to the change of the weir displacement. Equation (5) suggests that the sum of the three terms is equal to the inflow rate $q_{\text {in }}$, i.e., the conservation of fluid mass flow.

The equation for the weir bending subject to the hydrostatic pressure is described as follows.

$$
\rho^{\prime} \theta \ddot{s} / D+\partial^{4} s / \partial z^{4}= \begin{cases}\rho g(L-\eta) / D & {[0 \leqq z \leqq \eta]} \\ \rho g(L-z) / D & {[\eta \leqq z \leqq L]}\end{cases}
$$

For simplicity, we neglect inertia and assume that the external force is vertically uniform, $\rho g(L-\eta) / D$. Consequently, the above equation is simplified into the following equation,

$$
\partial^{4} s / \partial z^{4}=\rho g(L-\eta) / D
$$

with the following boundary conditions,

$$
\begin{aligned}
& s=\partial s / \partial z=0 \text { at } z=0, \\
& \partial^{2} s / \partial z^{2}=\partial^{3} s / \partial z^{3}=0 \text { at } z=L .
\end{aligned}
$$

Equation (7) with boundary condition ( 8 ) yields the following solution for the weir displacement :

$$
s(z)=\rho g(L-\eta)\left\{(z-L)^{4} / 4+L^{3} z-L^{4} / 4\right\} / 6 D .
$$

Integration of Eq. ( 9 ) from 0 to $L$ leads to the volume change of the upstream tank, $Q(t)$, due to the flexibility of the weir as follows:

$$
Q(t)=\int_{0}^{L} s(z) d z=\rho g(L-\eta) L^{5} / 20 D .
$$

\section{2 Instability condition}

The overflow height $h(t)$ is broken down into the equilibrium term $h_{0}$ and the time-dependent term $\Delta h(t)$ as follows :

$$
h(t)=h_{0}+\Delta h(t) .
$$

The overflow rate $q(t)$ is approximated by the first-order expansion by applying Eq. (11) to Eq. (4) as follows :

$$
q(t) \approx q_{0}+3 K h_{0}^{1 / 2} \Delta h(t) / 2 .
$$

Substituting Eq. (12) into Eq. (3), the springmass equation, Eq. ( 1 ) is rewritten in the following form : 


$$
\ddot{n}^{*}+c \dot{\eta}^{*}+k \eta^{*}=-3 A V\left(t_{0}\right) K h_{0}^{1 / 2} \Delta h(t-\tau) / 2 .
$$

In the same manner, Eq. ( 5 ) is rewritten in the following form, taking Eqs. (10) (12) into account: $\rho e(\Delta \dot{h}(t))+3 K h_{0}^{1 / 2} \Delta h(t) / 2-\rho^{2} g L^{5} \dot{\eta}^{*} / 20 D=0$.

Elimination of $\dot{\eta}^{* \prime} s$ by using Eqs. (13) and (14) leads to the following equation:

$$
\begin{aligned}
& \ddot{\eta}^{*}+k \eta^{*}= \\
& \quad-20 c D\left\{\rho e \Delta \dot{h}(t)+3 K h_{0}^{1 / 2} \Delta h(t) / 2\right\} / \rho^{2} g L^{5} \\
& \quad-3 A V\left(t_{0}\right) K h_{0}^{1 / 2} \Delta h(t-\tau) / 2 .
\end{aligned}
$$

Time integration of Eq. (15), from 0 to $T$, after multiplying $\dot{\eta}^{*}$, yields the following energy conservation equation :

$$
\begin{aligned}
& \dot{\eta}^{*}(T)^{2} / 2+k \eta^{*}(T)^{2} / 2-\left\{\dot{\eta}^{*}(0)^{2} / 2+k \eta^{*}(0)^{2} / 2\right\} \\
& \quad=-\int_{0}^{T} \dot{\eta}^{*}(t)[20 c D\{\rho e(\Delta \dot{h}(t)) \\
& \left.\quad+3 K h_{0}^{1 / 2} \Delta h(t) / 2\right\} / \rho^{2} g L^{5} \\
& \left.\quad+3 A V\left(t_{0}\right) K h_{0}^{1 / 2} \Delta h(t-\tau) / 2\right] d t .
\end{aligned}
$$

The left-hand-side terms in the above equation is the total mechanical energy (kinetic energy and potential energy) and the right-hand-side term represents the energy input from the water fall into the sloshing system in downstream tank during the time from 0 to $T$. Therefore, the instability condition is given as follows :

$$
\begin{aligned}
& -\int_{0}^{T} \dot{\eta}^{*}(t)[20 c D\{\rho e(\Delta \dot{h}(t)) \\
& \left.+3 K h_{0}^{1 / 2} \Delta h(t) / 2\right\} / \rho^{2} g L^{5} \\
& \left.+3 A V\left(t_{0}\right) K h_{0}^{1 / 2} \Delta h(t-\tau) / 2\right] d t>0 .
\end{aligned}
$$

The experimental observation indicates that the overflow height $h(t)$ and the downstream level $\eta(t)$ oscillate in almost the same phase at the early stage of the instability development. This fact allows us to approximate the two parameters as follows:

$$
\begin{aligned}
& \eta^{*}(t)=\varepsilon_{1} \sin \omega t, \\
& \Delta h(t)=\varepsilon_{2} \sin \omega t,
\end{aligned}
$$

where $\varepsilon_{1}$ and $\varepsilon_{2}$ are small positive constants. Since one cycle is long enough to examine the sign of integral (17), we use $2 \pi / \omega$ for $T$. An arithmetic manipulation after substituting Eqs. (18) and (19) into inequality

(17) leads to the following instability condition:

$\sin \omega \tau>40 \omega e D c h_{0} / 3 A V\left(t_{0}\right) q_{0} \rho g L^{5}$.

The above inequality reveals the meaningful features of fluid-elastic vibration of a flexible overflow weir: First, it is clear that the system is stable irrespective of lag-time, $\tau$, if the value of the right-hand side term exceeds unity. Therefore, it can be concluded that the increase in rigidity $D$ and angular frequency $\omega$ (or decrease of aspect ratio in our experiment) may suppress the instability. This coincides well with the experimental observation mentioned in section 2.2. Secondly, it is suggested that the increase in flow rate $q_{0}$ induces the instability, although no clear tendency of the flow rate dependency is experimentally observed. Thirdly, the system is always stable under any condition if $\sin \omega \tau$ is negative or if the lag-time satisfies the following condition.

$$
\pi / \omega+2 \pi N / \omega<\tau<2 \pi / \omega+2 \pi N / \omega \quad(N=0,1,2, \cdots)
$$

As seen in Figs. 4 (a) and (b), the vibrational instability in sloshing is not observed when the water level difference exceeds a certain value for each flow rate. On the other hand, inequality (21) indicates that the system becomes stable when the lag-time $\tau$ exceeds $\pi / \omega$, i.e., about $0.5 \mathrm{sec}$ for the present case. If the average speed of the water fall can be estimated at around $15 \mathrm{~cm} / \mathrm{sec}$, the critical water level difference, over which the sloshing system is stable, can be calculated at $7.5 \mathrm{~cm}$. Although the measurement of the lag-time for each water level difference was not conducted, the water level difference is directly related to the lag-time. These facts suggest that the experimental sloshing property is consistent with stability condition (21).

\section{3 Computational analysis of spring-mass model}

Since the above mathematical formulation is based on the perturbation analysis, direct time integration of the equation system was attempted to investigate the instability feature of the spring-mass system.

The spring-mass vibration of Eq. (4) with respect to the water elevation $\eta^{*}$ in the downstream tank is temporally integrated by the Newmark- $\beta$ scheme. The balancing equation of flow rate in the upstream tank, Eq. ( 5 ), is temporally integrated by the explicit Euler scheme to calculate the overflow height $h$ in the next time step. This computational procedure is repeated at every time step for the specified time interval unless computational instability occurs.

The direct time integration of the equations of the spring-mass vibration system is conducted using the parameter values described in Table 1. Figure 6 shows the typical instability development for the case of $A=$ $10 \mathrm{~m} / \mathrm{kg}$ and $\Delta t=0.1 \mathrm{sec}$. The instability development is very sensitive to the values of $A$ and $\Delta t$ because of the numerical viscosity introduced in the time integration scheme. Therefore, the value of $A$ is chosen to be unphysically large in the computation in order to overcome the numerical damping effect.

Secondly, the effect of the water level difference on the instability is numerically examined. The range of the water level difference used in this study is from $0.01 \mathrm{~m}$ to $0.22 \mathrm{~m}$, fixing $A$ at $4.0 \mathrm{~m} / \mathrm{kg}$ and $\Delta t$ at 0.05 sec. Figure 7 shows the peak-to-peak value of freesurface sloshing of water in the downstream tank 
against the water level difference, obtained by computation as well as experiment. It should be noted that no instability is observed in the following range of water level difference in the numerical result :

$0.06 m<L-\eta_{0}<0.15 m$.

The analytical stability condition (21) is specifically expressed for the parameters cited in Table 1 as :

$0.5 \mathrm{sec}<\tau<1.0 \mathrm{sec}$

Multiplying the average falling water velocity $V(=0.15 \mathrm{~m} / \mathrm{sec})$, the analytical stability condition (21) in terms of water level difference can be derived as follows :

$$
0.075 m<L-\eta_{0}<0.15 m
$$

From Eqs. (22) and (24), it is confirmed that the stability ranges predicted by the numerical calculation and analytical method are in fairly good agreement,

Table 1 Parameter values used for direct time integration

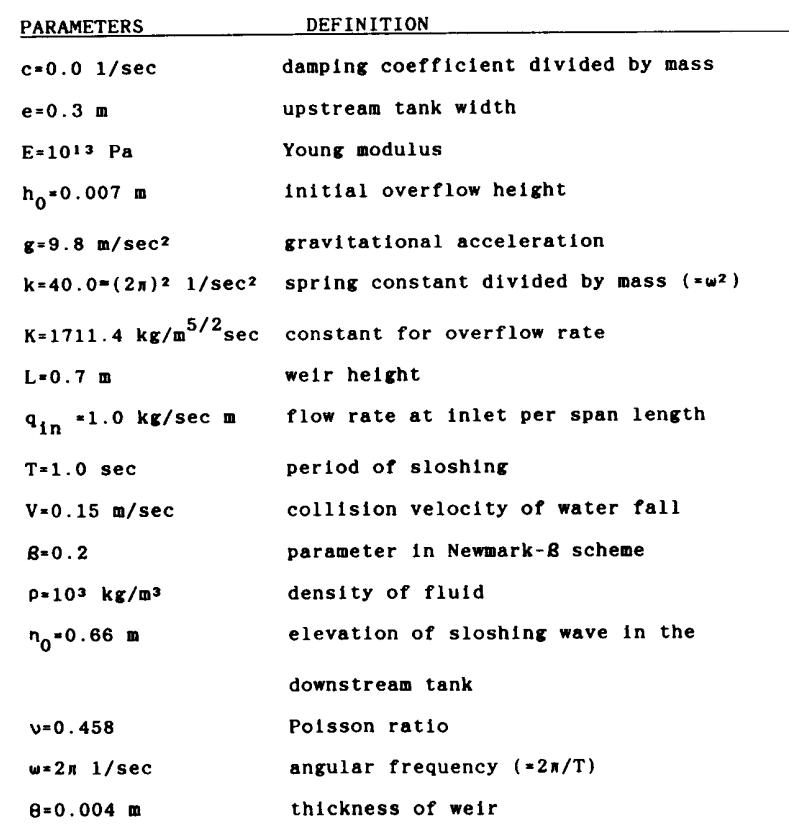

which justifies the validity of the derivation of the analytical instability condition. The comparison of this numerical result with the experimental one was not done due to the lack of experimental data, since the large water level difference (i.e., larger than 0.1 m) caused the weir structure to be destroyed due to the hydrostatic pressure.

\section{Discussion}

In the present study, the experimental and theoretical studies were done to clarify the fundamental feature of the fluid-elastic vibration of a flexible overflow weir. The experimental results have indicated that the instability occurs under a specific condition of the water level difference. The experimental results show that too rigid weir of $15 \mathrm{~mm}$ thickness did not allow for the instability but that the dependence of the rigidity on the instability is rather weak as long as the weir is flexible, i.e., 2 to $8 \mathrm{~mm}$ in thickness. It was also found in the experiment that the effects of the flow rate and the aspect ratio of the downstream tank were not very significant.

The analytical instability condition is derived by a perturbation method for the spring-mass model system, and it shows that the analytical instability

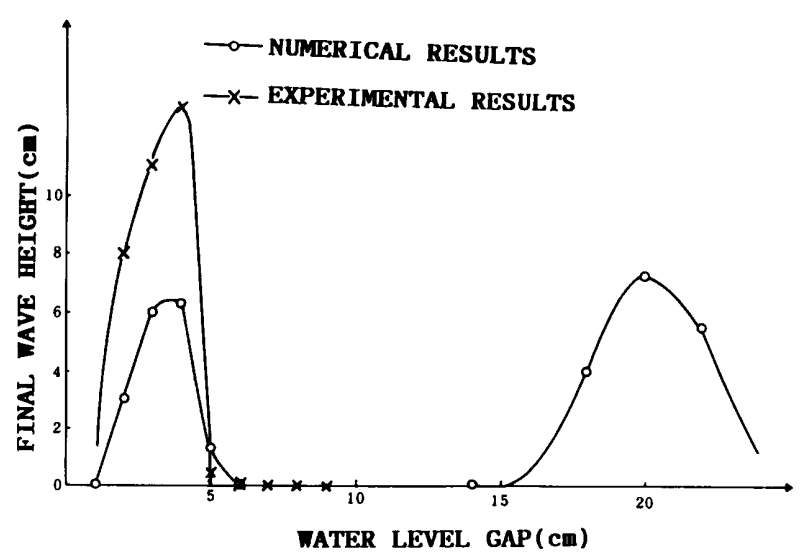

Fig. 7 Computational and experimental results of dependency of the instability on water level gap

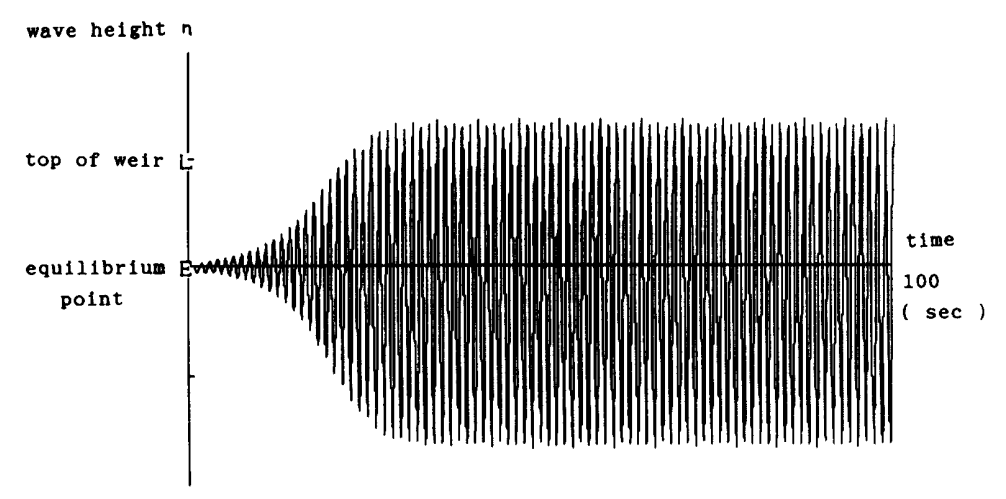

Fig. 6 Computational result of temporal sloshing evolution ( $L:$ height of weir $t_{\mathrm{op}}=70 \mathrm{~cm} . E:$ initial elevation $=88 \mathrm{~cm}$ ) 
condition explains most of the fundamental features observed in the experiment. The fundamental features obtained by the experiment, the mathematical analysis and computational analysis are fairly consistent with each other. These studies reveal that the instability is explained by the following six key steps.

STEP 1: The level of water free surface in the downstream tank is changed by the fluctuation of the overflow rate.

STEP 2: The change of the level of water free surface in the downstream tank moves the flexible weir.

STEP 3: The volume change of the upstream tank is induced by the displacement of the flexible weir.

STEP $4:$ The overflow rate is changed according to the level change of water free surface in the upstream tank.

STEP $5:$ The collision momentum of the overflow is changed according to the change of the overflow rate.

STEP 8: The mechanical energy in the downstream tank increases if the lag-time to travel from the top of the weir to the free surface satisfies a specific condition for phase difference of the collision momentum and the sloshing.

Instability will take place when the above six steps are repeated and the mechanical energy in the system is accumulated.

Aita et al. ${ }^{(2)}$ have pointed out that instability of the sloshing in a downstream tank appears when the flow rate and water level difference are rather small, while the other instability mechanism of a strong fluid-structure interaction (FSI) appears when the flow rate and the water level difference are large. The present instability mechanism is similar to that of the sloshing type, since the instabillty appears at small water level difference and the frequency is identical to the sloshing frequency of the downstream tank. The FSI was not observed in the present experiment, since the experimental apparatus used did not allow a large water level difference. The present spring-mass model is also restricted to the analysis of the sloshing-type instability, since the dynamic effect of the weir is neglected by eliminating the time-dependent inertia term in the plate bending vibration equation.

\section{Conclusions}

The fluid-elastic vibration of a flexible overflow weir placed in a rectangular tank was studied by experiment and theoretical analysis using a simple spring-mass model. It was found that the results obtained in the experiment are mostly explained by the mathematical and computational methods using a spring-mass model. Some important hydraulic parameters influencing the fluid-elastic vibration instability are identified by the experimental and theoretical studies, and it is found that the lag-time and water level difference are the most influential parameters among them.

\section{References}

(1) Aita, S., Tigeot, Y. and Gibert, R.J., "Fluid-elastic instability on Super Phenix LMFBR weir system : a survey", Proc. 9th Int. Conf. on Structural Mechanics in Reactor Technology (SMiRT), Lausanne, Vol. A (1987), p. 101.

(2) Aita, S., Tigeot, Y., Bertaut, C. and Serpantie, J. P., "Fluid-elastic instability in a flexible weir: experimental observations", Flow-Induced Vibration-1986, ASME PVP Conf., Vol. 104 (1986). p. 41.

( 3 ) Aita, S. and Gibert, R. J., "Fluid-elastic instability in a flexible weir: a theoretical model", FlowInduced Vibration-1986, ASME PVP Conf., Vol. 104,1986 , p. 51.

(4) Aita, S., Benassis. T., Gibert, R.J. and Gullbaud, D., "Fluid-elastic instability of a flexible weir: Nonlinear analysis" , Proc. Int. Conf. on Flow Induced Vibrations, Bowness-on-Windermere, England, 1987. p. 317.

(5) Lacroix, C. and Viollet, P. L., "Hydro-elastic study of instability of the cooling circuit of Superphenix" , Proc. IAEA specialist meeting on Flow Induced Vibrations in Fast Breeder Reactors, Paris, 1986.

(6) Lacroix, C. and Viollet, P. L., "Hydro-elastic study of instability of a crested weir to vibrations", traduction du rapport HE/44/86-24 (1987), National Laboratory of Hydraulics, Chatou, France.

(7) Hisano, K. and Kaneko, S., "Self-excited oscillation due to the fluid discharge over a flexible weir (1st report, excitation mechanism of a sloshing mode)" , Trans. JSME, Vol. 55, No. 511C, 1989, p. 541.

(8) Hisano, K. and Kaneko, S., "Self-excited oscilla tion due to the fluid discharge over a flexible weir (2nd report, excitation mechanism of a hydroelastic mode)" , Trans. JSME, Vol. 56, No. 525 C, 1990, p. 1094.

(9) Fukuie, M. and Hara, F., "Experimental study of self-excited vibrations of a flexible weir due to fluid discharge" , Trans. JSME, Vol. 55, No. 517 C, 1989 , p. 2282. 